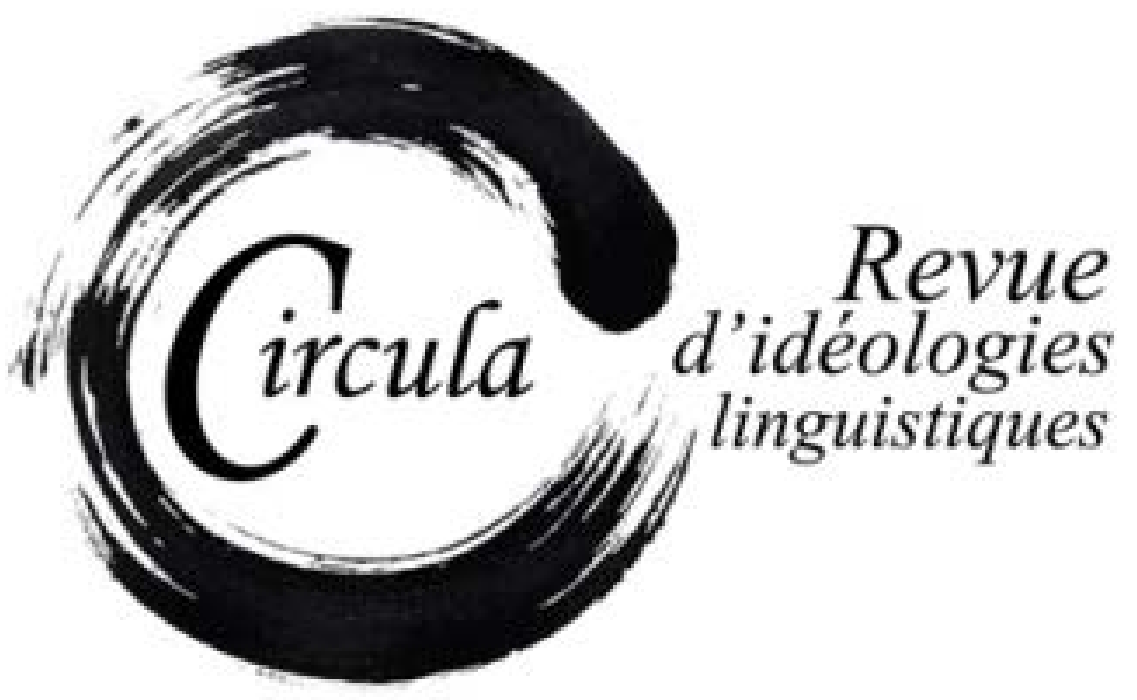

TITRE: «COME STIAMO A LINGUA? ... RISPONDE IL LINGUISTA». LA DIVULGAZIONE DEL SAPERE LINGUISTICO NELLE CRONACHE LINGUISTICHE FRA GLI ANNI 1950 E IL DUEMILA

Auteur(s): Sabine SchWARZE, Universität Augsburg

Revue: CirCula, NUMÉRO 5

PAGES: $108-131$

ISSN: 2369-6761

Directeurs: Wim Remysen, Sabine SCHWARZE et JuAn Antonio EnNiS

URI: HTTP://HDL.HANDLE.NET/11143/11235

DOI: HTTPS://DOI.ORG/10.17118/11143/11235 


\title{
«Come stiamo a lingua? ... Risponde il linguista». La divulgazione del sapere linguistico nelle cronache linguistiche fra gli anni 1950 e il Duemila
}

\author{
Sabine Schwarze, Universität Augsburg \\ sabine.schwarze@philhist.uni-augsburg.de
}

Riassunto: II contributo riguarda la retorica dei testi divulgativi su questioni di lingua pubblicati nella stampa italiana nell'arco di un periodo in cui si sono avverate modificazioni sostanziali a livello della norma standard e dell'uso linguistico nelle sfere discorsive alte e pubbliche. Il campione dei testi selezionati come base empirica proviene da cronache linguistiche e cioè da rubriche dedicate dai quotidiani a commenti critici, informativi o anche istruttivi sull'uso «corretto o adeguato» della lingua italiana, e firmate da specialisti nel campo (letterati, filologi, linguisti). Si propone essenzialmente un confronto di due cronache pubblicate fra gli anni 1950 e il primo decennio del Duemila in due dei più rinomati quotidiani nazionali, La Stampa e La Repubblica.

Il corpus è indagato con l'obiettivo di individuare le strategie retoriche adoperati dagli autori per esaminare i riflessi dei paradigmi scientifici che muovono la ricerca linguistica nel discorso divulgativo sulla lingua e la pertinenza di particolari tradizioni discorsivi nella scelta di tali strategie.

Parole chiave: divulgazione; tradizioni discorsive; cronaca linguistica; strategie retoriche

Abstract: This paper deals with the rhetoric of articles about language related topics published in Italian newspapers in a period of substantial changes in the field of the standard norm and the language use in the high and public discourse spheres. The sample of texts which serves as an empirical basis comes from language columns which provide critical, informative or instructive comments on the "correct or adequate" use of the Italian language, texts which were signed by specialists in the field of literature, philology and linguistics. We compare two language columns published in two of the most renowned national daily newspapers, La Stampa and La Repubblica, between the 1950s and the first decade of the 21st century. The aim of this comparison is to identify rhetorical strategies adopted by the authors to examine both the reflection on scientific paradigms which have been the subject of linguistic research on the popular discourse on language and the relevance of specific discourse traditions for the selection of these strategies.

Keywords: vulgarization; discourse traditions; language columns; rhetorical strategies 


\section{Introduzione}

Il seguente contributo riguarda la retorica dei testi divulgativi nell'ambito dei mass media. Il campione dei testi selezionati come base empirica proviene da cronache linguistiche ${ }^{1}$, dedicate dai quotidiani a commenti critici, informativi o anche istruttivi sull'uso «corretto o adeguato» della lingua italiana, e firmate da specialisti nel campo (letterati, filologi, linguisti). Si propone essenzialmente un confronto di due cronache pubblicate fra gli anni 1950 e il primo decennio del Duemila in due dei più rinomati quotidiani nazionali, La Stampa e La Repubblica. II corpus è indagato con l'obiettivo di individuare le strategie retoriche adoperate dagli autori per esaminare la pertinenza di particolari tradizioni discorsive nella scelta di tali strategie e i riflessi dei paradigmi scientifici che muovono la ricerca linguistica nel discorso divulgativo sulla lingua. In una prospettiva contrastiva saranno perciò analizzati il metalinguaggio e la topica degli articoli, la costruzione di autorità nel dialogo con i lettori e la struttura dei titoli.

\section{La divulgazione del sapere linguistico}

\subsection{Linguistica laica vs. linguistica scientifica}

L'interpretazione dei discorsi metadiscorsivi sulla lingua, come si avverano nelle cronache linguistiche, ha portato alla discussione sulla natura del sapere linguistico da essi veicolato. I concetti che variano fra 'linguistica popolare', 'linguistica laica' o anche 'linguistica ingenua' hanno in comune la distinzione di tale campo dalla linguistica detta «scientifica» oppure «accademica». Se in ambito italofono gli studi mettono il focus piuttosto sull'espressione del sapere linguistico «inconscio» oppure ancora «ingenuo», parlando ad esempio come Sgroi (2010) di una «grammatica laica»² o ancora come Graffi di «morfologia e sintassi ingenua» ${ }^{3}$ ciò corrisponde in linea di massima all'uso etnolinguistico della nozione di folk linguistics creata molto prima in ambito anglosassone ${ }^{4}$.

1. La nostra definizione di cronaca linguistica segue la nozione di chroniques du langage proposta già in Verrault (2007) e ampliata in seguito nei lavori di Remysen (2005 e 2009).

2. Nell'obiettivo di interpretare l'analisi linguistica effettuata dal parlante (comune e non esperto) Sgroi distingue fra tre tipi di grammatica, vale a dire (1) la «grammatica immanente della comunità» (sistema aperto di regole costitutive acquistato da colti e incolti in modo inconsapevole); (2) la «grammatica inconscia o mentale» (sottosistema individuale posseduto dal singolo parlante) e (3) la «grammatica teorica» (sistema elaborato e studiato a scuola e quindi più ristretto rispetto al primo tipo; cf. Sgroi (2010).

3. Nell'accezione di Graffi comprendono l'uso intuitivo di nozioni che fanno riferimento all'interpretazione della sintassi senza essere fondate su principi o approcci espliciti, cf. Graffi (1994: 25-33).

4. Il termine è stato coniato nell'ambito dell'antropologia linguistica negli anni 1960 da Hoenigswald e ampliato sin dagli anni 1980 da Preston e altri, cf. fra gli altri Hoenigswald (1966) e Niedzielski/Preston (2000); per la traduzione tedesca Volkslinguistik in termini di «tutti gli enunciati ritenuti di espressione naturale [...] riferendosi a fenomeni linguistici o funzionando a livello della metacomunicazione» [trad. it. S. Schw.], cf. Brekle (1989: 39). 
In prospettiva meramente didattico-divulgativa è stato coniato invece in ambito germanofono il termine Laienlinguistik (linguistica laica) che intende una linguistica normativa dei manuali di conversazione o di espressione orale, destinati a migliorare le competenze linguistiche dei parlanti nella vita sociale e professionale:

Laien-Linguistik bezeichnet eine Sprach- und Kommunikationsbetrachtung für Laien und häufig genug auch eine, die von Laien betrieben wird. Der Begriff Laien-Linguistik deckt sich dabei in weiten Teilen mit dem, was man »normative« oder »präskriptive Linguistik« nennen könnte. Sie umfaßt aber mehr: deskriptive, enzyklopädisch ausgerichtete und/oder unterhaltende Darstellungen zu sprachlich-kommunikativen Themen oder Problemen. (Antos, 1996:

$\left.25^{5}\right)$

In ambito francese gli studi rivolti alla «linguistique populaire» rimangono scarsi fino ai primi anni del Duemila. Il concetto di linguistica popolare adoperato poi dagli studiosi sembra escludere la divulgazione del sapere linguistico per mettere il focus su «tout type d'activités métalinguistiques destinées aux non experts à distinguer d'une popularisation des résultats de la linguistique dite 'scientifique'»(Paveau, 2005: 96)'.

Nell'ambito della comunicazione mediatica la cronaca linguistica, genere giornalistico predestinato alla diffusione di sapere linguistico fra un pubblico di non esperti, può avvalersi di funzioni multiple? Ciò non esclude però il suo uso a scopo divulgativo specie quando - come nel caso della maggior parte delle cronache linguistiche pubblicate nella stampa italiana - gli autori sono, a prescindere da poche eccezioni, dei professionisti.

\subsection{Il discorso divulgativo e l'utenza nella knowledge society}

La definizione generica di divulgazione come diffusione della conoscenza con lo scopo di «suscitare curiosità e partecipazione in un pubblico [di non esperti] molto differenziato per livello educativo, competenze, interessi» (cf. Angela, 2009) al giorno d’oggi richiede senz'altro una specificazione per quanto stiamo in una fase di accrescimento non solo dell'utenza cui si trasmette il sapere ma anche

5. Linguistica laica denomina un approccio alla lingua e alla comunicazione per non esperti e spesso anche avanzato dagli stessi non professionisti. Il concetto include grosso modo quello che si intende con linguistica "normativa" o ancora "prescrittiva". Include però anche altro: la presentazione descrittiva, in chiave enciclopedica oppure dilettevole di argomenti e problemi linguistico-comunicativi [trad. S. Schw.]. Cf. Antos (1996: 25).

6. In tale senso il campo della linguistica popolare a matrice francese riprende e include altri concetti già ampiamente discussi in Francia come immaginaire linguistique e activités épilinguistiques, cf. fra gli altri Houdebine (2002) e Canut (1998).

7. V. ad esempio Schwarze (1977: 21-33). 
degli emittenti di esso ${ }^{8}$. Tale processo coincide sui formati della divulgazione scientifica come anche sul carattere del sapere veicolato.

I formati di divulgazione linguistica proposti dal giornalismo (non scientifico) che qui c'interessa, avvertono da alcuni anni un superamento del classico modello della comunicazione unidirezionale (one to many) per proporre9, ad esempio con i blog o forum, formati screen to face bidirezionali e interattivi ${ }^{10}$.

Ci si dovrebbe quindi interrogare sul carattere del sapere e sui modi di costituzione e legittimazione della conoscenza scientifica in ambito linguistico. Seguendo due sociolinguisti francesi, la sempre crescente disponibilità del sapere con l'uso delle nuove tecnologie avrebbe provocato «un certain effacement des différences entre professionnels du savoir (que sont les universitaires par exemple) et détenteurs profanes de savoirs ou de savoirs profanes» (Achard-Bayle/Paveau, 2008 : 4). Gli stessi autori propongono giustamente di interpretare la scientificità di un discorso non in base al binarismo scientifico/popolare o profano ma su una scala graduale:

La linguistique populaire pose un problème de frontières disciplinaires et de conception de la science. Sur ce point les questions se bousculent: quels sont les rapports entre sociolinguistique et linguistique populaire (intégration, affinité, croisement) ? Et surtout entre linguistique populaire et linguistique dite savante ou scientifique : faut-il rester sur une opposition binaire en "vs" ou, plus raisonnablement, poser les choses en termes de continuum, de gradient de scientificité ou spontanéité? (Achard-Bayle/Paveau, 2008: 7)

Sulla stessa scia, nell'introduzione a un volume che raccoglie alcuni saggi sul destino del tedesco e dell'italiano come lingue scientifiche, Sobrero evidenzia una «modularità [progrediente] di ogni lingua speciale» (Sobrero, 2006: 5) in funzione di un'utenza sempre più vasta e differenziata, cui bisognerebbe adeguare le scelte linguistiche e testuali per ogni tipo di discorso specialistico. Secondo la classificazione usuale nella linguistica settoriale, sarebbero da distinguere nella dimensione verticale almeno tre categorie, vale a dire il discorso fra esperti, il discorso semi-divulgativo e quello divulgativo. Sobrero va ancora oltre e mette in rilievo come la scienza si adegua sempre di più alle leggi

8. L'impatto delle nuove tecnologie comunicative su tale processo è discusso in un testo programmatico pubblicato dall'UNESCO: "The new information and communication technologies have created new conditions for the emergence of knowledge societies. Added to this, the emerging global information society only finds its raison d'être if it serves to bring about a higher and more desirable goal, namely the building, on a global scale, of knowledge societies" (cf. UNESCO, 2005). Il concetto di società del sapere riprende in parte quello di società d'informazione per intendere 'una società in cui si generalizzano la diffusione e l'uso (il consumo) della conoscenza in base all'uso delle tecnologie d'informazione e di comunicazione a basso costo a scopo della disseminazione del sapere e della creatività.

9. In una prospettiva diacronica sarebbe più lecito interpretare tali formati come un ritorno al dialogo, già favorito come formato di divulgazione in altre epoche, dal dialogo filosofico di Platone attraverso il dialogo rinascimentale fino al dialogo in epoca dei Lumi. Cf. fra gli altri Kalverkämper (1989: 17-80).

10. Per la divisione della comunicazione in tre macro-categorie e cioè la comunicazione face to face (one to one), la comunicazione dei mass media (one to many) e la comunicazione screen to face (one to one, one to many, many to many) cf. Di Bari (2010: 122). 
del mercato: «La lingua dei testi scientifici arriva sul mercato, oggi, come un'auto: in tante versioni quante sono le tipologie degli utenti che si vogliono raggiungere» (Sobrero, 2006: 5).

L'interpretazione dei discorsi linguistici divulgativi dovrebbe prendere in considerazione inoltre le tradizioni discorsive che determinano il settore disciplinare, a livello particolare di una comunità linguistica come a livello generale della comunità scientifica in determinati periodi. Da un lato, la scienza stessa non costituisce un campo omogeneo ma consta di un gran novero di discipline dotate di una cultura specifica. Dall'altro lato, le lingue in quanto riflessi di tradizioni intellettuali e culturali hanno sviluppato delle tradizioni discorsive a loro specifiche. Se una gran parte degli studiosi ritiene funzionale un sistema di norme metodologiche, linguistiche e etiche universali, da tempo è stato osservato invece che tali norme dipendono dalle specifiche condizioni storiche in cui gli scienziati si inseriscono ${ }^{11}$.

Per quanto riguarda il discorso scientifico italiano, occorre senz'altro ricordare il conflitto risultante dall'influsso persistente del linguaggio poetico-letterario, inserito in una tradizione letteraria secolare e «immutabile» e vantato per la sua flessibilità e stabilità, conflitto ancora persistente in epoca moderna nella discussione sulla famosa scissione fra le cosiddette «due culture», umanistica e scientifica. In veste d'esempio si potrebbe ricordare l'ormai famoso dibattito fra due dei massimi esponenti, Carlo Bernardini e Tullio De Mauro, con la citazione del rimprovero avanzato al linguista di collegare sempre l'erudizione all'eleganza della lingua: «questo "vostro" pensiero che bada solo a essere erudito ed elegante e non si preoccupa minimamente del rigore semantico, può avere responsabilità enorme nella formazione dell'uomo contemporaneo» (Bernardini/De Mauro, 2003: 6)12.

Seguendo gli studi a proposito della divulgazione scientifica si potrebbero riassumere alcune strategie generali da rispettare da parte degli autori:

- Adoperare una lingua comprensibile: strategia che riguarda la terminologia, il metalinguaggio, la chiarezza e l'efficacia degli enunciati;

- Coinvolgere il lettore: strategia che riguarda l'uso di elementi dialogici, la creazione di identità collettiva;

- Attirare l'interesse (dilectare), motivare l'utenza: strategia che riguarda la valorizzazione, l'emozionalizzazione e anche l'insistenza del discorso;

- Consolidare l'argomentazione: strategia che riguarda il riferimento ai fatti dell'uso linguistico e alle autorità tramite citazioni, esempi, norme/regole.

11. Sulla base di un concetto idealizzato della scienza concepita come «riflesso della verità», almeno fino agli anni 1980 l'esistenza di uno 'stile scientifico universale' non veniva contestata. Un approccio relativistico e basato sulla specificità culturale delle singole discipline in dipendenza dalla cultura linguistica di base vi si oppone in modo più sistematico dalla metà degli anni 1990.

12. Per un commento più dettagliato cf. anche Schwarze (2007: 261-274). 
- Mettere a disposizione del lettore dei riferimenti scritti: strategia che riguarda il riferimento a opere teoriche, dizionari, grammatiche, manuali, testi scritti modelli e/o scrittori/scriventi modello).

\section{La cronaca linguistica come genere divulgativo in Italia}

\subsection{La divulgazione del sapere linguistico come attività dei professionisti}

Se c'è qualcosa che i linguisti stranieri invidiano ai colleghi italiani, è la risonanza che da noi i problemi linguistici hanno presso il gran pubblico: non esiste altro paese nel quale le pubblicazioni di divulgazione linguistica abbiano un mercato altrettanto ampio, i temi di linguistica godano della stessa audience nei mass-media, i linguisti (o almeno alcuni linguisti) possano vantare analoga notorietà. Linvidia non riguarda tanto lıaspetto economico della situazione [...], quanto la possibilità di diffondere anche fuori dell'ambito specialistico il sapere acquisito dalla ricerca. (Cortelazzo, 1991: 29)

Seguendo Cortelazzo, l'attività divulgativa sembra essere di spiccato interesse fra i linguisti italiani che intervengono - più che in altri paesi - anche nei mass media quando si tratta di problemi di lingua. A prescindere da una lunga tradizione risalendo oltre il momento della fondazione delle prime testate nazionali a uso comune, nella knowledge society globalizzata di oggi la discussione sui fenomeni legati ad usi linguistici nella comunicazione quotidiana gode di un mercato massmediatico molto vivace ${ }^{13}$.

Si tratta con la lingua senz'altro di un tema onnipresente e pertinente per tutti i membri delle comunità linguistiche, siano essi esperti e o non esperti. Va tuttavia sottolineato un fattore che distingue il campo della divulgazione linguistica da altri campi di divulgazione del sapere disciplinare: nella società odierna, i non-professionisti in materia linguistica dispongono di una formazione scolastica più o meno approfondita, che include alcuni strumenti (anche terminologici e concettuali) per l'interpretazione dei sistemi linguistici. Anche se il parlante comune non ha lo stesso tipo di specializzazione del linguista, non è quasi mai del tutto incompetente in materia. In più, spesso e tradizionalmente, la socializzazione scolastica in ambito linguistico è legata a una visione in cui «a lingua è (o dovrebbe essere) oggetto statico, separato dai parlanti, e meritevole di conservazione» (Santulli, 2015: 55). ̇̀

13. Tanto più stupisce il fatto che al giorno d'oggi bibliografie e studi empirici italiani nel campo della linguistica laica o popolare, prima del 2013 erano praticamente inesistenti. L'unico studio più sostanzioso che riportò anche alcuni risultati empirici fu pubblicato in lingua tedesca nel 2007 e non ebbe diffusione in Italia (cf. Demel, 2007; preceduta da un riassunto diacronico, sempre in lingua tedesca, sul discorso metalinguistico popolare che include anche le cronache linguistiche, cf. Demel, 2006). 
coniata perciò da ideologie trasmesse da secoli, come l'ideologia del purismo linguistico e della lingua standard, basate su modelli letterari e arcaici ${ }^{14}$.

Il linguista che interviene pubblicamente sul suo oggetto di lavoro si deve porre il problema del comportamento da tenere per scegliere secondo Cortelazzo (co-autore lui stesso di una cronaca linguistica) essenzialmente fra due alternative [corsivo S. Schw.]:

[...] riproporre il sano atteggiamento scientifico di semplice osservatore, ed interpretatore, della realtà linguistica, o gettarsi nell'arena e, forte delle sue conoscenze di situazioni linguistiche del passato e di regole di buon funzionamento della lingua, rendersi paladino di un determinato uso linguistico da far emergere dalla gamma di abitudini diverse ed anche contrastanti che convivono nell'attuale momento di transizione della lingua italiana? (Cortelazzo, 1991: 29)

Nelle cronache linguistiche mediate dalla stampa, le strategie retoriche sono funzionali a tale scelta per variare fra un modo istruttivo-prescrittivo e uno informativo-descrittivo. Confrontando l'asse diacronico e sincronico diventano trasparenti le oscillazioni fra i due poli, interpretabili in funzione ai paradigmi scientifici dominanti, ma nello stesso tempo anche in funzione alle ideologie linguistiche favorite dalla società.

\subsection{La stampa come luogo di divulgazione e il corpus}

La stampa italiana non ha mai cessato, sin dalla nascita delle riviste letterarie nel Settecento, di commentare i problemi derivati dalla Questione della lingua (Qdl) e di pubblicare, spesso in serie, degli articoli che avevano come tema le norme linguistiche. Spesso organizzati in cronache o rubriche linguistiche, gli articoli delle testate nazionali e anche regionali hanno contribuito a formare a modo loro i rapporti che gli italiani hanno stabilito con la loro lingua. Dopo l'unificazione politica e per lo più dagli anni 1950 tali cronache s'impegnavano a rafforzare la standardizzazione dell'uso formale scritto. Se in quegli anni del dopoguerra si nota un «decisivo decremento del registro aulico e letterario, in linea con la tendenza generale secondo la quale la letteratura non rappresenta ormai più un modello o un punto di riferimento per nessun tipo di scrittura», nella maggior parte dei quotidiani

14. Esistono pochi studi sulla tipologia delle ideologie linguistiche. Si potrebbero individuare con Maitz (2014) sette ideologie linguistiche popolari: (1) Decadentismo linguistico (storia linguistica interpretata come storia di una corruzione, degradazione; (2) Omogenismo linguistico (variabilità linguistica non è desiderata, bisogna invece coltivare il modello unico della buona lingua); (3) Difettismo linguistico (esistono nella lingua e nelle sue varietà forme di per se difettose e dannose); (4) Purismo linguistico (le risorse autoctone della lingua valgono più di qualsiasi prestito straniero); (5) Elitismo linguistico (l'uso linguistico dei ceti sociali alti e colti supera di qualità quello dei ceti sociali bassi); (7) Standardismo (la varietà standard supera di qualità il non-standard). Nella posizione neutra descrittiva che determina l'ottica dello scienziato vi si oppongono almeno tre ideologie professionisti in relazione ai paradigmi disciplinari determinanti per la linguistica contemporanea: 1) Liberalismo (cambiamenti interpretati come processi naturali nel corso della storia di una lingua); 2) Pluralismo (variabilità naturale e utile, diversità come ricchezza); 3) Egalitarismo (tutela e valorizzazione di tutte le variazioni linguistiche). 
permane il «gradimento per una lingua sostenuta e sorvegliata», con un lessico difficile e lontano dalla lingua parlata di quei lettori che la stampa vorrebbe tanto raggiungere (cf. Bonomi, 2002: 4243). D’altro canto, forse dovuto proprio alla presenza mediatica di studiosi di lingua e letteratura, la stampa mantiene la tradizione di piattaforma per il dibattito linguistico nel paese.

Se il ruolo dell'Italia come caso modello per la diffusione di sapere linguistico scientificamente consolidato attraverso i mass media (stampa, radio e televisione) è stato variamente dimostrato ${ }^{15}$, la figura del professionista di lingua in veste di cronista richiede tuttavia una precisione. Per la situazione italiana si rivela significativa la forte presenza, anche dopo l'essersi consolidata la figura del giornalista professionista, di personaggi noti dell'ambiente accademico, di letterati e filologi. A dimostrare uno spiccato interesse per i problemi di lingua erano inoltre i critici cinematografici datosi che nella questione linguistica la lingua filmica occupa un posto notevole ${ }^{16}$. È emblematico il caso di Leo Pestelli (1909-1976), autore fra il 1953 e il 1976 di ben cinque cronache e ancora oggi citato non di rado quando si tratta di riflettere sull'uso corretto e appropriato della lingua italiana. Ha svolto attività come giornalista nell'ambito della critica letteraria e cinematografica e come scrittore. Come linguista sarebbe da attribuire al gruppo di autori che rientrano nel campo della linguistica laica destinata a migliorare le competenze linguistiche dei parlanti ${ }^{17}$. Altri autori noti di cronache linguistiche svolgono delle attività accademiche d'impronta filologica (come Bruno Migliorini, Tristano Bolelli, Giorgio de Rienzo e Gian Luigi Beccaria) oppure più strettamente sociolinguistica ${ }^{18}$ (come Tullio de Mauro, Michele Cortelazzo e Massimo Arcangeli).

15. Cf. fra gli altri Ernst (1998, pp. 210-211) e Schmitt (2001, pp. 482-484). Tanto più sorprende lo scarso interesse che gli studiosi abbiano manifestato (a parte poche eccezioni) per il ruolo della stampa scritta come luogo della circolazione di ideologie linguistiche e per il suo influsso sulla coscienza linguistica e sull'imaginario linguistico degli italiani oppure sul processo di normalizzazione dell'italiano. Questa situazione si potrebbe spiegare, in parte, come riflesso del fatto che in Italia, molto più della stampa, i media audio-visivi (radio, cinema e innanzitutto la televisione) avevano, nella storia recente dell'unificazione linguistica, assunto il ruolo di «scuola dell'italiano».

16. Rossi parla in proposito di «una vocazione metalinguistica e metaculturale» del cinema italiano, da lui discussa di recente sulla base di materiale raccolto nelle cronache linguistiche pressoché ignote di cinque riviste specializzate negli anni Trenta-Quaranta, cf. Rossi (2015, p.31-32).

17. Lo dimostrano oltre alle sue cronache, alcune pubblicate anche in formato di libro, varie opere di carattere linguistico-filologico a scopo meramente didattico-divulgativo che ebbero una discreta diffusione: Parlare italiano con diverse edizioni fra il 1957 e il 1979; Trattatello di rettorica. Contro l'anarchismo e la tecnocrazia trasportati nella lingua con diverse edizioni fra il 1969 e il 1985 e il Dizionario delle parole antiche pubblicato varie volte fra il 1961 e il 1990.

18. Si intende qui la sociolinguistica moderna portando sulle interrelazioni fra lingua e società e metodologicamente instauratasi come tale «nel corso degli anni Sessanta, in connessione da un lato con gli studi sulla rilevanza del linguaggio nell'educazione del sociologo Basil Bernstein in Gran Bretagna e dall'altro lato, e soprattutto, con le ricerche sui correlati sociali della variazione linguistica di William Labov in America»; per un riassunto, anche sull'instaurarsi della sociolinguistica in Italia, cf. Berruto (2011). 
Per il presente contributo sono state selezionate due cronache linguistiche pubblicate in periodi diversi e firmate da professionisti della lingua italiana ${ }^{19}$. La cronaca Come stiamo a lingua (in seguito CSL) pubblicata dal quotidiano torinese La Stampa fra il 01/08/1953 e il 25/05/1955 in terza pagina consta di 75 articoli firmati dal noto critico letterario e cinematografico Leo Pestelli20. La cronaca /l linguista (in seguito IL) prende spunto sull'iniziativa di Massimo Arcangeli (curatore della rivista scientifica Lid'O. Lingua italiana d'oggi) il 2 giugno 2009 sulla La Repubblica online nel settore «Giovani e scuola» ed è tuttora attiva. Vi partecipano autori vari, tutti professionisti in ambito linguistico, con articoli su problemi dell'uso linguistico cui si aggiunge alcune volte l'anno un appello all'utenza di porre delle domande ${ }^{21}$.

\section{Alcuni risultati di un’analisi contrastiva delle due cronache}

\subsection{Topica, metalinguaggio e criteri valutativi}

La terminologia linguistica adoperata rimanda certo agli strumenti canonici della descrizione linguistica (in riferimento alle grammatiche e ai dizionari) ma nell'insieme il metalinguaggio utilizzato assume un ruolo più complesso e non solo descrittivo. La scelta particolare si muove sostanzialmente riguardo al grado di professionalità dell'utenza avvisata, alla comunità scientifica con il rispettivo paradigma scientifico in cui l'autore si inserisce e all'ideologia linguistica dell'autore, spesso trasmessa negli elementi valutativi (che possono riguardare gli enunciati o la natura stessa della lingua).

I criteri valutativi rivelano essenzialmente due ideologie linguistiche diverse in cui si riflette il paradigma scientifico predominante. Nella cronaca degli anni Cinquanta risultano nettamente predominanti i criteri valutativi trasportati nella riflessione sulla lingua italiana sin dall'epoca della sua prima normalizzazione. Anche se l'autore riconosce i cambiamenti avvenuti come processi naturali nel corso storico della lingua italiana, in questo «liberalismo» traspare tuttavia l'ideologia del decadentismo con qualche ambizione di «risanare questa benedetta lingua»:

19. Gli articoli sono stati selezionati dal corpus CROMit in allestimento presso la cattedra di linguistica romanza dell'Università di Augsburg. La raccolta consta attualmente di 33 cronache linguistiche pubblicate fra il 1951 e il 2014.

20. Pestelli vi cura complessivamente cinque cronache i cui titoli sembrano indicare un atteggiamento profondamente ancorato nell'ideologia puristica della lingua (nazionale): La lingua pura e impura (1956-1961); Difesa della lingua (19611968) seguita da Difesa della lingua italiana (1969-1973). Negli articoli l'autore si astiene invece anche esplicitamente dal purismo linguistico e dai «pedanti» e si dimostra più liberale nei confronti dei cambiamenti di lingua ritenuti anche da lui «inevitabili».

21. La cronaca offre quindi un servizio di consulenza «in tempo reale»: lancia regolarmente un appello agli utenti a comunicare agli autori i loro «dubbi sulla grammatica», cf. Dubbi 2015. 
Lo strazio che oggi si fa delle particelle, è di quelle cose che il linguista non si sente di perdonare. [...] [la preposizione a] È forse la voce più maltrattata del vocabolario, il «souffre-douleur» del cattivo parlar moderno. [...] e bene farebbe quel trattore che non desse ascolto a codeste barbare ordinazioni e volesse prima rovinarsi la clientela che l'udito. (CSL, 19 settembre $1953)^{22}$

L'autore usa una to pica tradizionale che risale alla storica polemica con i francesi fra Sei e Settecento e che riguarda la qualità della lingua:

I diminutivi e in genere i nomi sono un privilegio della lingua italiana [...] Non avrebbe invece potuto amarli di più il Tommaseo, come «quelli che attestano la pieghevolezza e la soavità della lingua, e quella ricchezza che vorrei dire morale, cioè accomodata ai delicati bisogni del sentimento». Verissimo [... Certo vanno usati con discrezione e a proposito. (CSL, 29 settembre 1953)

Anche prendendo distanza da posizioni meramente puristiche coltivate nell'Ottocento come la condanna di francesismi, nell'accettazione di parole o enunciati entrati ormai nell'uso, l'autore include avvolte una critica esplicita del loro scarso valore estetico. Tale critica può culminare in un giudizio di stampo chiaramente puristico, nel dare conclusivamente «torto all'uso». Ciò dimostra la discussione sull'uso di «indomani» già ampiamente discusso nell'Ottocento ${ }^{23}$.

L'indomani ci viene dalla lingua francese che lo possiede ab antiquo: dapprima coll'articolo diviso dal nome, poi come agglutinazione [...]. Volendo imitare bene, dovemmo scrivere lindomani, maniera che ci parrebbe mostruosa, mentre l'altra non lo è meno, abbiamo così fatto l'orecchio da non sentirci più né il barbaro né il ricercato. Concludendo, considerato come sia facile dire il giorno dopo o, classicamente, il domani, noi ancora una volta, da queste noterelle per antiquari della lingua, daremo torto all'uso. (CSL, 17 ottobre 1953)

II riferimento a criteri estetici della lingua traspare invece solo poche volte e piuttosto in funzione ornamentale che argomentativo nella cronaca curata da linguisti odierni come in un bando di concorso lanciato in collaborazione con IL nel 2009 (S.O.S. Salviamo le nostre belle parole, IL, Massimo Arcangeli, 22 settembre 2009) ${ }^{24}$ oppure in riferimento a gusti dell'utenza di fronte ai quali il linguista prende una posizione neutra (una «femminilizzazione» della professione che fa storcere il naso anche a molte donne, IL, Massimo Arcangeli, 3 giugno 2009). Vi prevalgono invece chiaramente argomenti

22. Gli articoli analizzati si citano dalla versione digitalizzata già integrata nel corpus CILIt dove sono classificati con le indicazioni seguenti: autore, giornale, titolo cronaca, data. Nelle citazioni seguenti si indicano la sigla della cronaca e la data della pubblicazione. A differenza dalle citazioni tratte da opere scientifiche la citazione degli esempi si mette in corsivo.

23. Cf. ad esempio la cronaca «Note di lingua», pubblicata a firma di Giuseppe Rigutini su La Domenica Letteraria dal 1882 al 1885 di recente studiata da Valentina Allia, cf. Allia in questo numero di Circula.

24. Siccome si tratta di una cronaca firmata da autori vari, nella citazione degli esempi si indicano la sigla della cronaca, la data e l'autore. 
pragmatici a favore della funzionalità comunicativa dei segni linguistici prendendo in considerazione la variazione interna della lingua che sostituisce il modello unico e omogeneo. La posizione correttiva e prescrittiva cede chiaramente a quella descrittiva come illustra in esplicito il seguente brano sulla femminilizzazione delle professioni:

L'ipotesi più economica, [...], è in definitiva agire per ora sugli unici fattori che sopportano, senza alcuna difficoltà, la declinazione al femminile dei nomi coinvolti: i determinanti articolativi e attributivi. Dirò allora non solo la soprano e la contralto, senza che la regola patisca eccezioni, ma anche la sindaco e la questore, oppure una magistrato e una chirurgo. Accordi, me ne rendo conto, che potrebbero provocare rimostranze "puriste" o suscitare proteste da parte di molte donne. A me paiono però risolvere salomonicamente l'impasse di fronte ai casi nei quali la femminilizzazione non è ancora stata pienamente accolta nell'uso. Il quale, I'ho scritto più volte, è comunque sovrano. A nulla varrebbe tentare di opporgli una qualche resistenza, che ci piaccia o no. (IL, Massimo Arcangeli, 3 giugno 2009)

La valutazione pragmatica dei termini risalta anche nella descrizione di formule di saluto in italiano che distingue i diversi registri con usi adeguati e validi:

Come si salutano, oggi, gli italiani? Nel linguaggio corrente il portabandiera delle formule di saluto, anche in virtù di una tendenza diffusa all'uso di un registro informale, è l'amichevole ciao, che si utilizza all'inizio e alla fine di un incontro fra persone che si danno del tu. Termine fortunatissimo perché breve, incisivo, facile da pronunciare con un sorriso. (IL, Simonetta Losi, 14 giugno 2009)

Il mutamento degli approcci linguistici (accettazione di registri adeguati all'uso in determinate situazioni e con determinati interlocutori, affronto neutro di continui mutamenti in particolare nel lessico) diventa oggetto di interi paragrafi d'impronta teorica. Così un articolo sui neologismi recenti contiene un riassunto dettagliato sull'interpretazione del forestierismo nel dibattito sulla lingua italiana per concludere con il «rigetto di teorie puristiche e neopuristiche» nella linguistica moderna:

La linguistica moderna ha rigettato le teorie puristiche e neopuristiche, puntando a farsi osservatrice dei fenomeni linguistici e rinunciando a ogni forma di coercizione: in altre parole, la "linguistica prescrittiva" ha ceduto il passo alla "linguistica descrittiva". L'eco di un mutato orientamento culturale è ravvisabile anche nel campo dei neologismi, che vengono oramai studiati senza preconcetti, nonché con strumenti di analisi adeguati. (IL, Debora de Fazio e Rocco Luigi Nichil, 19 ottobre 2009)

Si avverte però, all'interno della rubrica, anche un atteggiamento più «normativo» (nel senso della linguistica laica più strettamente intesa). Lo si nota in un articolo che propone dopo una definizione assai semplificata di norma («si può forse definire la norma linguistica come uno strumento con il quale individuare ciò che è linguisticamente accettabile. E con il quale, addirittura, stabilire una gra- 
dualità: quanto e come è accettabile una certa forma? La norma ce lo dice. E ci dice anche che cos'è un “errore”») una spiegazione alquanto generica di ‘purismo' basata su un approccio tradizionale:

\section{Purismo e non-purismo}

Ogni parlante, e non solo grammatici e scrittori, può scegliere di essere più o meno purista; a seconda che sia più propenso a respingere le nuove forme dettate dall'uso (purista), oppure ad accettarle (non-purista). Più i parlanti si rendono conto della complessità dei fenomeni linguistici, più sono consapevoli della difficoltà di distinguere nettamente ciò che è accettabile da ciò che non lo è. Diverso è il caso dei grammatici, che adottano consapevolmente l'uno o l'altro atteggiamento in base a una tradizione più o meno fedele ai modelli letterari canonici. (IL, Yahis Martari, 26 giugno 2009)

Una chiara distinzione fra linguisti e non «addetti ai lavori» si nota anche a livello metalinguistico nell'articolo «La linguistica» di Fausto Raso. Stilisticamente si avverte l'esperienza cospicua nel campo della linguistica laica e della scrittura «blogistica», a cominciare dalla denominazione dell'articolo come «noterella» ${ }^{25}$. Ne citiamo un breve estratto:

I non "addetti ai lavori" quando sentono parlare di linguistica storcono il naso e pensano a un argomento noioso e non degno di essere preso in considerazione, e tutt’al piú si limitano a dire che è "un qualcosa che riguarda la lingua". Queste modeste noterelle si prefiggono lo scopo - se possibile - di avvicinare anche i piú riottosi allo studio (o, se preferite, alla conoscenza) di questa meravigliosa scienza. (IL, Fausto Raso, 30 agosto 2013)

Segue una descrizione semplificata delle diverse sottodiscipline della linguistica per concludere con una captatio benevolentia che richiama l'equalità che secondo l'autore dovrebbe assumersi un testo divulgativo e cioè:

Non sappiamo, francamente, se siamo riusciti nell'intento prefissoci: di avvicinare alla lingua anche i piú restii. Ma tant'è. Abbiamo cercato di essere concisi al massimo per non appesantire il tutto sforzandoci, nel contempo, di essere chiari. Se non ci siamo riusciti confidiamo nel vostro... "perdono". E concludiamo citando una massima di Ferdinand de Saussure: «Il segno linguistico unisce non una cosa e un nome, ma un concetto e un'immagine acustica». (IL, Fausto Raso, 30 agosto 2013)

Vanno comunque anche menzionate le tracce di un metalinguaggio in cui traspare chiaramente l'ideologia di decadentismo dalla quale i linguisti comunemente si astengono. In un titolo Difendiamo il congiuntivo (IL, Fausto Raso, 18 giugno 2009) l'uso del tradizionale metalinguaggio bellico sembra comunque indicare un atteggiamento condiviso, in particolare quando si tratta di un «tema 'scot-

25. Raso è iniziatore di alcuni blog su problemi linguistici mirati sulla scrittura mediatica d'impronta decisamente più didattico prescrittiva ma stilisticamente anche più «giornalistico» e dilettevole. Il suo blog Lo SciacquaLingua. Noterelle sulla lingua italiana. Per coloro che amano il bel parlare e il bello scrivere nel titolo non solo riporta un topos tradizionale ma cita con Noterelle sulla lingua italiana anche il titolo di una delle cronache del Pestelli. 
tante' (il nostro bel congiuntivo, troppo spesso dimenticato o maltrattato)» che suscita tuttora vivi dibattiti anche fra linguisti.

\subsection{La costruzione d'autorità: il rapporto fra autore-esperto e pubblico-non-esperto}

Strettamente legato al comportamento scelto dall'autore - il «sano atteggiamento scientifico di semplice osservatore, e interpretatore, della realtà linguistica» oppure la difesa di un determinato uso linguistico da «paladino» (cf. Cortelazzo, 1991: 29) - sarà anche il rapporto che costruisce con il lettore e la definizione più o meno esplicita della ripartizione dei rispettivi ruoli.

Ben consapevole del rapporto asimmetrico fra un autore il cui imaginario linguistico è saldamente ancorato alla tradizione letteraria classica e un pubblico sul quale la letteratura e il modello letterario della lingua non avrebbero più esercitato influenza notevole, Pestelli cerca di conquistare il lettore e di legittimare la sua autorità come esperto di lingua mettendo in atto diverse strategie complementari. Assume una posizione alquanto critica nei confronti dell'uso moderno della lingua italiana senza porre però ferrea resistenza ai mutamenti in corso. I numerosi riferimenti intertestuali in cui si riproducono discorsi precedenti dei Classici della letteratura italiana oppure dei teorici della lingua ${ }^{26}$. Consensuali o contrari alla sua posizione tali riferimenti intertestuali servono a dimostrare la competenza professionale dell'autore, ma nello stesso tempo avvertono anche l'assenza di presunzione d'infallibilità. Sin dal primo articolo Pestelli si astiene dall'invocare interventi normativi sulla lingua. A favore del buon senso comune e del buon gusto, richiamati come criteri valutativi dell'uso, rispinge sistematicamente l'idea del potere autoritario di grammatici e accademie per mettere in rilievo il genio linguistico dello scrittore:

Dai Classici si può imparare non soltanto l' osservanza delle regole grammaticali, ma anche e soprattutto la violazione di esse; imparare quel che il De Amicis, nel suo bel libro sulla lingua, chiama «gli ardiri» e poi spiega, umoristicamente come «la cravatta per traverso». Alle regole si deve il rispetto che alle vecchie zitelle, condito d' un interno risolino circa le loro virtù supposte. [...] (CSL, 1 agosto 1953)

Varie volte si astiene anche in modo esplicito da posizioni puristiche rigide (escludendosi chiaramente dai «pedanti» della norma) come ad esempio nella discussione intorno alla sostituzione di loro da gli:

Metteteci tutti i loro che i pedanti vorrebbero, e avrete un guazzabuglio. Loro urta male anche coi pronomi lo, la, le: conosciuta la fanciulla chiamai i parenti e la restituii loro; molto meglio: ... e gliela restituii. In molti casi si può dunque usare gli per loro, massime nel linguaggio

26. Prevalgono riferimenti ad autori e grammatici Ottocenteschi, e quindi al modello linguistico promosso dopo l'unificazione e con la progrediente scolarizzazione degli italiani, come Tommaseo, Fanfani, Rigutini, De Amicis (L'idioma gentile) e Collodi (Pinocchio). 
famigliare o quando il buon suono o la naturalezza del costrutto lo chiedano; e in nessuno mai l'usarlo è vergogna. (CSL, 14 novembre 1953)

Dall'altro lato adopera uno stile brillante, mai asciutto ed essenziale ma pieno di effetti retorici, battute spiritose, ironia, citazioni che mette in rilievo tutta la seduzione di una lingua "sintatticamente immacolata, ricca di parole calzanti e univoci" che non si rispecchierebbe più negli usi da lui commentati27. Riesce in quel modo a sdrammatizzare la sua distanza dal pubblico come vediamo nel brano successivo:

Se la grammatica ci fosse di casa, non diciamo come il catechismo, ma soltanto come il settimanale illustrato, non avverrebbe che l'uscita al plurale di certi nomi pur familiarissimi, ci tenesse così perplessi da doverne interrogare il primo che passa, o peggio, una cattedra come questa nostra. E ancora questi scrupolosi sono un piccolo numero rispetto ai moltissimi che ignari del problema lo risolvono pazzamente da sè. (CSL, 24 ottobre 1953)

Spesso e volentieri Pestelli distingue le competenze linguistiche del suo pubblico in base allo stato civile e sociale supponendo una stratificazione ben netta. Il dialogo con i lettori di sesso femminile (cui più che ad altri si perdonano licenze linguistiche) illustra che abbiamo a che fare con un periodo precedente al political correctness.

Uno degli errori più perdonabili, specie in bocca a donna coniugata; anzi un errore che dota la sposa, è lo scambio del modo congiuntivo col modo indicativo. «Bisogna che mi alzo...». «Vuoi che lo faccio?», sono solecismi da fare impallidire in società; ma pronunziati fra le pareti domestiche, effondono pace, attestano d'uno spirito sereno, innamorato della certezza. $\mathrm{Ci}$ sono donne, anche discretamente istruite, che al congiuntivo usciranno, a far molto, un paio di volte il giorno: nature colombine cui le cose si presentano di faccia, contente al quia, che non danno al marito altro pensiero che di procurar loro il becchime. (CSL, 22 agosto 1953)

Nonostante faccia valere la sua autorità linguistica, Pestelli si intende meno consulente che non osservatore critico della decadenza della sua lingua di cui denuncia i colpevoli. La lingua stessa, presentata come un organismo vivo che percorre diverse fasi evolutive di vitalità ma anche di malattia, diventa un «interlocutore» maltrattato da una gran parte della comunità linguistica. L'autore esprime la sua «compassione» con battute spiritose e metafore biologiche.

Il nostro linguaggio è un intarsio d'arti e mestieri; ma con palesi ingiustizie, perchè ci sono voci che fanno i servizi più disparati, che sgobbano per sette. Organizzare è una, organizzandosi così un esercito, le scuole, uno spettacolo, una partita di canasta (e ordinare, disporre, allestire ecc., stanno a guardare); [...] non canzona neppure il sostantivo manifestazioni, comprensivo e di spettacoli e di convegni e di gare sportive e di congressi scientifici; una parola

27. Si cita dal necrologio a Leo Pestelli che tenta di imitare il suo stile presentandolo come «buongustaio dell'idioma gentile» che «consigliava e ammaestrava» i lettori «senza alcun piglio cattedratico», cf. Colombo (1976). 
così sovraccarica che per compassione le si è regalato un quadro: il quadro delle manifestazioni. Anche esibire, [...] oltredichè, violentata dall 'uso, ha figliato i mostricini esibizionismo ed esibizionista, i quali non hanno bisogno di spiegazione. (CSL, 5 settembre 1953)

La cronaca pubblicata online in formato di blog prevede un servizio interattivo. IL è strutturato come vero e proprio dialogo con i lettori come dimostra bene il lancio regolare dell'appello a comunicare con i curatori del blog28.

Dubbi sull'italiano? Risponde il linguista/7

Avete dubbi sulla grammatica? Scriveteli in questo forum e un gruppo di linguisti risponderà in tempo reale a ogni vostra singola domanda.

Un servizio di consulenza dedicato soprattutto agli studenti in ansia per la maturità ma aperto a chiunque voglia sollevare questioni sulla nostra lingua. Le risposte, a cura della redazione di “Lid’O. Lingua italiana d’oggi”, sono coordinate dal linguista Massimo Arcangeli.

Indirizzo permanente del post / 194 commenti

Con il pubblico si istaura un rapporto di quasi-simmetria (cui corrisponde anche la scelta di un registro più informale e giovanile) e gli si riconosce una certa formazione linguistica (il pubblico avvisato consta prevalentemente di studenti della scuola secondaria). Ogni tanto all'utente viene perfino attribuita una certa autorità linguistica che si esprime ad esempio nel suo coinvolgimento nel risolvere problemi come la selezione di neologismi (con la «notoria fantasia italica» ma anche con l'aiuto del buon senso comune):

Assai più della "regolatezza" (leggi: funzionalità) poté il genio. A imporsi, fra le centinaia di proposte di traduzione di blog, chat, newsletter, provider, spamming, i fervidi frutti della notoria fantasia italica. Digidiario, blabele, infolettera, telefornitore, digiluvio postale: queste, a nostro giudizio, le cinque migliori soluzioni prodotte dai partecipanti al concorso lanciato da Repubblica.it, con la collaborazione dell'editore Zanichelli. [...] (IL, Massimo Arcangeli, 16 agosto 2010)

Un cambiamento verso un "giornalismo» decisamente partecipativo si può avvertire anche nella presa di distanza dell'autore dell'articolo seguente dalle autorità (accademiche?). A «qualche notabile della lingua» (l'autorità del quale viene messa in dubbio tramite virgolette) si attesta «l'usanza deleteria»:

Si va sempre più affermando l'usanza deleteria - introdotta da qualche "notabile della lingua" - di sostituire il congiuntivo con l'indicativo; così (dice il "notabile") tutto si semplifica e i bambini (bontà sua) non trascorreranno le notti insonni per "capire" la differenza che intercorre tra i due modi del verbo. Non si può però pretendere di fare scomparire un modo di un

28. L’appello è stato lanciato sette volte, si cita dall'ultimo pubblicato il 15 aprile 2015, cf. Dubbi 2015. 
verbo adoperato da secoli per enunciare un fatto come incerto, possibile, sperato e del cui esito, perciò, non si è sicuri; diversamente dall'indicativo, che esprime la certezza o la realtà constatata (o immaginata) nella nostra mente come tale. (IL, Fausto Raso, 18 giugno 2009)

\subsection{Struttura e funzione dei titoli}

Passiamo infine all'analisi di un elemento strutturale di rilievo per la cronaca linguistica in quanto genere giornalistico. Per la stampa il titolo delle rubriche e dei singoli articoli rappresenta il primo elemento di contatto con il lettore. Nella scrittura scientifica ha la funzione di riassumere il contenuto del testo cui si riferisce. Nella stampa invece il titolo «può essere considerato un microtesto che condensa l'informazione, ma rappresenta soprattutto un invito alla lettura, tanto da poter essere descritto come la pubblicità dell'articolo» (Santulli 2015, p. 61)29. Sarà interessante indagare perciò se gli autori fanno uso di strategie retorico-persuasive per motivi «promozionali» o se prevale la funzione del riassunto abbreviato a scopo informativo.

Dal confronto delle due cronache appare che gli autori ricorrono nella struttura dei titoli a tre strategie principali: 1) l'enunciazione del tema con funzione di anticipazione del contenuto; 2) Informazione incompleta e/o implicita sul contenuto con funzione retorico-persuasivo; 3) Gioco sul rapporto fra parole e cose/persone rappresentate.

\section{(1) L'enunciazione del tema con funzione di anticipazione}

Il primo tipo (una combinazione della brevitas con la perspicuitas) si avverte piuttosto marginale nella cronaca di Pestelli, vi troviamo tuttavia titoli come Parole nuove, dubbi nuovi (CSL, 24 aprile 1954), La regola grammaticale e l'esempio degli scrittori (CSL, 23 novembre 1954) o Parole che non sono più (CSL, 13 novembre 1954).

Si tratta invece del tipo prevalente nella cronaca /l linguista. Accanto alla semplice indicazione del tema trattato come Salutarsi in italiano (IL, Simonetta Losi, 14 giugno 2009), Come ti ringrazio (e ti ricambio) (IL, Simonetta Losi, 21 giugno 2009) o Punto, due punti, punto e virgola... (IL, Fabio Ruggiano, 10 luglio 2009) vi troviamo anche titoli con ambizione teorica come Dalla teoria alla prassi: norma e uso (IL, Yahis Martari, 26 giugno 2009), La linguistica (IL Fausto Raso, 30 agosto 2013) in cui si utilizza anche la terminologia linguistica come Anàfora e catàfora (IL, Fausto Raso, 11 giugno 2014).

29. Nella sua analisi del dibattito sull'italiano che focalizza fra le altre anche la struttura dei titoli degli articoli scelti, Santulli indica come studi recenti sull'argomento di particolare interesse Bell (1992) e Held (1999). 


\section{(2) Informazione incompleta e/o implicita sul contenuto con funzione retorico-persuasivo}

Retoricamente più interessante è invece un secondo tipo che dà una informazione incompleta e/o implicita sul contenuto. In tali casi la brevitas si combina con una carenza di perspicuitas per far diventare il titolo uno strumento per la cattura dell'attenzione. Il titolo invita implicitamente alla lettura, necessaria per completare il quadro informativo solo confusamente tracciato. Come già evidenziato da Santulli nel saggio sopra menzionato si tratta nello stesso tempo di una richiesta di accordo con il lettore che comporterebbe «una affermazione etica ma anche il riconoscimento di oggetti comuni su cui fondare l'accordo stesso» (cf. Santulli 2015).

Nella cronaca di Pestelli, i titoli spesso rispecchiano elementi caratteristici del discorso linguistico tradizionale (e tradizionalistico). Si notano alcuni residui della polemica secolare con la cultura linguistica francese, cf. Parole "parvenues" e parole decadute (CSL, 21 agosto 1954). L'autore ricorre spesso alla personalizzazione delle parole, come ad esempio con Lamento di un pronome (CSL, 23 ottobre 1954) o Prepotenze contro i piccoli (19 settembre 1953). Pestelli istaura uno stretto rapporto fra uso linguistico e posizione sociale nei titoli che collegano all'uso "corretto" della lingua un comportamento adeguato nella società per bene, cf. Quello che deve sapere la fidanzata per bene (CSL, 26 settembre 1953). Spesso i titoli ironizzano su usi linguistici «sbagliati» e le loro possibili conseguenze, cf. «Sciolto» il dubbio del marito qualcosa ci rimane tuttavia (CSL, 24 ottobre 1953) o Signora in beige e marito al verde (CSL, 6 novembre 1953). Possono anche riportare su usi ormai fuori moda ma rimpianti dall'autore, cf. Fra sua figlia e me ci è convenienza di umori (CSL, 31 ottobre 1953).

Nella cronaca nata in epoca telematica si avvertono, più frequentemente negli articoli dei linguisti particolarmente coinvolti e presenti nei mass media, titoli del secondo tipo che adoperano strategie retoriche tipiche per la scrittura mediatica. Un titolo come L'accento val bene una messa (giusta) (IL, Massimo Arcangeli, 2 giugno 2009) mette in gioco la polisemia di messa e l'identificazione di un'espressione ormai di carattere formulare, trasportata nella memoria culturale italiana fino ad oggi, e originaria dalle famose parole «Parigi val bene una messa» con cui Enrico IV avrebbe rinunciato alla fede protestante in favore di quella cattolica pur di conquistare il regno di Francia. La stessa strategia si avverte per un sottotitolo come Assai più della "regolatezza" (leggi: funzionalità) poté il genio, frase introduttiva di un altro articolo che il lettore italiano difficilmente legge senza ricordare il famoso verso del XXXIII canto dell'Inferno di Dante (IL, Massimo Arcangeli, 16 agosto 2010).

\section{(3) Titoli che giocano sul rapporto fra parole e cose/persone rappresentate}

Alcuni titoli giocano sul rapporto fra parole e referenti reali, senza chiarire se si tratta dell'uno o dell'altro come in "II Ministro" o "Ia Ministra"? Fra i due litiganti... (IL, Massimo Arcangeli, 3 giugno 2009) oppure in strutture più esplicite che indicano l'opponente fra parentesi come La (parola) reginetta dell'anno. Quale sarà (IL Massimo Arcangeli, 29 ottobre 2009), Vince la (parola) crisi (IL, Massimo Arcangeli, 30 gennaio 2010). 
Sono significativi i titoli del secondo e terzo tipo per la funzione d'intrattenimento che senza dubbio va spesso attribuita alla discussione su usi linguistici nei mass media. Anche se i contenuti cambiano nel tempo in quel quadro comunicativo l'autore capta l'interesse dell'utenza non solo con la sua competenza professionale ma anche con la retorica non strettamente scientifica.

\section{Conclusione}

Se la posizione di Pestelli come autore di cronaca linguistica negli anni 1950 è meramente segnata da posizioni ideologiche, basate sull'apprezzamento di qualità estetiche della lingua e sulla qualifica degli usi linguistici in relazione all'appartenenza sociale dei locutori (con la terminologia di Cortelazzo, si comporta da «paladino» dell'italiano aulico tradizionale), al giorno d'oggi prevalgono delle posizioni «tecniche» oppure pragmatiche, basate sulla descrizione ragionata del sistema linguistico, delle sue difficoltà e irrazionalità. Tale mutamento è senz'altro collegato all'arco di una cinquantina di anni che corre fra le due cronache analizzate. Si tratta di un periodo in cui si sono avverate modificazioni sostanziali a livello dell'uso linguistico nelle sfere discorsive pubbliche ma anche nei paradigmi scientifici dominanti della linguistica.

Il materiale non permette tuttavia un'interpretazione lineare come passaggio da linguistica prescrittiva (sulla base di un concetto omogeneo e stabile di lingua) a linguistica descrittiva e neutra (sulla base di un concetto variazionale di lingua con i suoi correlati sociali). II cronista degli anni Cinquanta percepisce senz'altro la necessità d'evoluzione linguistica della sua comunità e concede la massima autorità all'uso. Ciononostante rimane ancorato nell'imaginario linguistico fortemente impregnato di valori estetici e letterari e lo fa valere.

La distanza notevole fra l'autorità modello dell'autore (come anche delle referenze citate) e l'autorità dell'uso di altri attori massmediatici e degli utenti che ancora si avverte nella cronaca CSL sembra essere decisamente ridotta nella cronaca IS. Mentre nella cronaca degli anni 50 l'autore non di rado adopera il ruolo del maestro, nel caso della cronaca pubblicata online un pubblico «linguisticamente più maturo» partecipa (certo anche stimolato dai dispositivi interattivi forniti dagli strumenti web) alla soluzione di problemi linguistici. Nella sua massima forma (blog, forum ecc.) questa interattività consiste in una specie di upgrade del lettore emancipato. Internet ha dunque contribuito a sviluppare un giornalismo partecipativo di cui si avvale anche nelle cronache linguistiche in quanto favorisce un taglio discorsivo più adatto alle esigenze comunicative degli utenti di oggi.

Una citazione conclusiva, in cui si commenta la proposta di un utente di sostituire l'anglicismo blog dall'espressione italiana fluario con la suggestione di un neologismo (ludico?) da parte del linguista, illustra sia il carattere dialogico della scrittura divulgativa mediatica odierna sia la sua funzione dilettevole: 
Quanto al blog, [...] Molto originale fluario, giustificato così: "Mentre un diario è qualcosa di immutabile, statico, una semplice foto del mondo scattata da un particolare punto di vista, un blog è una creatura vivente, in trasformazione, fatta per interagire ed arricchirsi". Più per interagire o per arricchirsi? Direi, se stiamo al gioco, per interarricchirsi. (IL, Massimo Arcangeli, 16 agosto 2010) 


\section{Bibliografia}

\section{Fonti primari}

\section{Articoli citati da CSL = Come stiamo a lingua}

Pestelli, Leo (1953), «I vespisti coi loro crepiti sono nati senza levatrice», La Stampa, 1 agosto 1953, p. 3.

Pestelli, Leo (1953), «l baffi del gendarme», La Stampa, 22 agosto 1953, p. 3.

Pestelli, Leo (1953), «Dal tuono alla zanzara», La Stampa, 5 settembre 1953, p. 3.

Pestelli, Leo (1953), «Prepotenze contro i piccoli», La Stampa, 19 settembre 1953, p. 3.

Pestelli, Leo (1953), «Quello che deve sapere la fidanzata per bene», La Stampa, 26 settembre 1953, p. 3.

Pestelli, Leo (1953), «Cattivo sangue fra i puristi», La Stampa, 17 ottobre 1953, p. 3.

Pestelli, Leo (1953), "Sciolto" il dubbio del marito qualcosa ci rimane tuttavia», La Stampa, 24 ottobre 1953, p. 3.

Pestelli, Leo (1953), «Fra sua figlia e me ci è convenienza di umori», La Stampa, 31 ottobre 1953, p. 3.

Pestelli, Leo (1953), «Signora in beige e marito al verde», La Stampa, 6 novembre 1953, p. 3.

Pestelli, Leo (1953), «Le tiglie della notte ed i giovani di tatto», La Stampa, 14 novembre1953, p. 3.

Pestelli, Leo (1954), «Parole nuove, dubbi nuovi», La Stampa, 24 aprile 1954, p. 3.

Pestelli, Leo (1954), «Parole «parvenues» e parole decadute», La Stampa, 21 agosto 1954, p. 3.

Pestelli, Leo (1954), «Lamento di un pronome», La Stampa, 23 ottobre 1954, p. 3.

Pestelli, Leo (1954), «Parole che non sono più», La Stampa, 13 novembre 1954, p. 3.

Pestelli, Leo (1954), «La regola grammaticale e l'esempio degli scrittori», La Stampa, 23 novembre 1954, p. 3. 


\section{Articoli citati da IL = $\|$ Linguista}

Arcangeli, Massimo (2009), «L'accento val bene una messa (giusta)», La Repubblica, 2 giugno 2009, http://linguista.blogautore.repubblica.it/2009/06/02/l-accento-val-bene-una-messa-giusta/ [Sito consultato il 20 novembre 2015].

Arcangeli, Massimo (2009), «"Il Ministro" o "la Ministra”? Fra i due litiganti...», La Repubblica, 3 giugno 2009, http://linguista.blogautore.repubblica.it/ 2009/06/03/il-ministro-o-la-ministra-frai-due-litiganti/ [Sito consultato il 20 novembre 2015].

Arcangeli, Massimo (2009), «S.O.S. Salviamo le nostre belle parole», La Repubblica, 22 settembre 2009, http://linguista.blogautore.repubblica.it/2009/09/22/sos-salviamo-le-nostre-belle-parole/ [Sito consultato il 20 novembre 2015].

Arcangeli, Massimo (2010), «Vince la (parola) crisi», La Repubblica, 30 gennaio 2010, http://linguista.blogautore.repubblica.it/2010/1/30/vince-la-parola-crisi/ [Sito consultato il 20 novembre 2015).

Arcangeli, Massimo (2010), «Ci 6? Blabliamo? Rx x fav», La Repubblica, 16 agosto 2010, http://linguista.blogautore.repubblica.it/2010/08/16/che-si-fa-blabliamo/ [Sito consultato il 20 novembre 2015].

Arcangeli, Massimo (2015), «La (parola) reginetta dell'anno. Quale sarà», La Repubblica, 29 ottobre 2015, http://linguista.blogautore.repubblica.it/2015/10/29/la-parola-reginetta-quale-sara/ [Sito consultato il 20 novembre 2015].

De Fazio, Debora/Nichil, Rocco Luigi (2009), «Una passeggiata fra i neologismi del Terzo Millennio», La Repubblica, 19 ottobre 2009, http://linguista.blogautore.repubblica.it/2009/10/19/una-passeggiata-fra-i-neologismi-del-terzo-millennio/ [Sito consultato il 20 novembre 2015].

Dubbi sull'italiano? Risponde il linguista/7, http://linguista.blogautore.repubblica.it/2015/04/15/ dubbi-sullitaliano-risponde-il-linguista7/ [Sito consultato il 20 novembre 2015].

Losi, Simonetta (2009), «Salutarsi in italiano», La Repubblica, 14 giugno 2009, http://linguista.blogautore.repubblica.it/ 2009/06/14/salutarsi-in-italiano/ [Sito consultato il 20 novembre 2015].

Losi, Simonetta (2009), «Come ti ringrazio (e ti ricambio)», La Repubblica, 21 giugno 2009, http://linguista.blogautore.repubblica.it/ 2009/06/21/come-ti-ringrazio-e-ti-ricambio/ [Sito consultato il 20 novembre 2015].

Martari, Yahis (2009), «Dalla teoria alla prassi: norma e uso», La Repubblica, 26 giugno 2009, http:// linguista.blogautore.repubblica.it/2009/06/26/dalla-teoria-alla-prassi-norma-e-uso/ [Sito consultato il 20 novembre 2015].

Raso, Fausto (2009), «Difendiamo il congiuntivo», La Repubblica, 18 giugno 2009, http://linguista. blogautore.repubblica.it/2009/06/18/difendiamo-il-congiuntivo/ [Sito consultato il 20 novembre 2015]. 
Raso, Fausto (2013), «La linguistica», La Repubblica, 30 agosto 2013, http://linguista.blogautore. repubblica.it/2013/08/30/la-linguistica/ [Sito consultato il 20 novembre 2015].

Raso, Fausto (2014), «Anàfora e catàfora», La Repubblica, 11 giugno 2014, http://linguista. blogautore.repubblica.it/2014/06/11/anafora-e-catafora/ [Sito consultato il 20 novembre 2015].

Ruggiano, Fabio (2009), «Punto, due punti, punto e virgola ...», La Repubblica, 10 luglio 2009, http://linguista.blogautore.repubblica.it/2009/07/10/punto-due-punti-punto-e-virgola/ [Sito consultato il 20 novembre 2015].

\section{$\underline{\text { Studi }}$}

Achard-Bayle, Guy/Paveau, Anne-Marie (2008), «Présentation. La linguistique "hors du temple"», Pratiques. Linguistique, littérature, didactique, no 139-140: Linguistique populaire, p. 3-16, https://pratiques.revues.org/1171 [Sito consultato il 20 luglio 2017].

Angela, Piero (2009), «Le vie della divulgazione scientifica”, Enciclopedia Italiana, http://www.treccani.it/enciclopedia/le-vie-della-divulgazione-scientifica_(XXI-Secolo)/ [Sito consultato il 20 luglio 2017].

Antos, Gerd (1996), Laien-Linguistik. Studien zu Sprach- und Kommunikationsproblemen im Alltag. Am Beispiel von Sprachratgebern und Kommunikationstrainings, Tübingen, Niemeyer.

Beacco, Jean-Claude (ed.) (2004), « Représentations métalinguistiques ordinaires et discours », Langages, nº 154 .

Bell, Allan (1992), The Language of News Media, Oxford, Blackwell Publishers.

Bernardini, Carlo/De Mauro, Tullio (2003), Contare e raccontare. Dialogo sulle due culture, Roma/Bari, Laterza.

Berruto, Gaetano (2011), «Sociolinguistica», Enciclopedia Italiana, http://www.treccani.it/ enciclopedia/sociolinguistica/ [Sito consultato il 30 giugno 2017].

Bonomi, Illaria (2002), L'italiano giornalistico. Dall'inizio del '900 ai quotidiani on line, Firenze, Cesati.

Bourdieu, Pierre (2001), Langage et pouvoir symbolique, Paris, Seuil.

Brekle, Herbert (1989), «La linguistique populaire», in Auroux, Sylvain (ed.), Histoire des idées linguistiques, Bruxelles, Mardaga, p. 39-44.

Canut, Cécile (1998), «Pour une analyse des productions épilinguistiques, Linguistique et représentation(s) », Cahiers de praxématique, n³1, p. 69-90.

Clas, André et al. (1975-1976), Bibliographie des chroniques de langage publiées dans la presse du Canada, vol. 1 (1950-1970), vol. 2 (1879-1949), Montréal, Université de Montréal. 
Colombo, Furio (1976), «Morte improvvisa di Leo Pestelli. Umanista, critico del cinema, prezioso scrittore», La Stampa, 04 dicembre 1976, p.3.

Cortelazzo, Michele A. (1991), «Una lingua in movimento», Corriere del Ticino, 9 novembre 1991, p. 29, http://www.cortmic.eu/cronache/cronache002.html [Sito consultato il 31 maggio 2015].

De Mauro, Tullio (1998), Storia linguistica dell'Italia unita, Bari/Roma, Laterza.

Demel, Daniela (2006), «Laienlinguistik und Sprachchroniken: Italienisch», in Ernst, Gerhard et al. (ed.), Romanische Sprachgeschichte. Ein internationales Handbuch zur Geschichte der romanischen Sprachen, Berlin, de Gruyter, vol. 2, p. 1523-1532.

Demel, Daniela (2007), Si dice o non si dice? Sprachnormen und normativer Diskurs in der italienischen Presse, Frankfurt, Peter Lang.

Di Bari, Riccardo (2010), L'era della web communication. Il futuro è adesso, Trento, Tangram Edizioni Scientifiche.

Ernst, Gerhard (1998), «ltalienische Sprachkultur im Überblick», in Greule, Albrecht/Lebsanft, Franz (ed.), Europäische Sprachkultur und Sprachpflege, Tübingen, Narr, p. 210-211.

Graffi, Giorgio (1994): Sintassi, Bologna: Il Mulino, p. 25-33.

Held, Gudrun (1999), «Il titolo come strumento giornalistico», Études Romanes, n 42, p. 173-189.

Hoenigswald, Henry M. (1966), A proposal for the Study of Folk-Linguistics, in Sociolinguistics. Proceedings of the UCLA Sociolinguistic Conference 1964, a cura di W. Bright, The Hague, Mouton, p. 1626.

Houdebine, Anne-Marie (2002), L'imaginaire linguistique, Paris, l'Harmattan.

Kalverkämper, Hartwig (1989), «Kolloquiale Vermittlung von Fachwissen im frühen 18. Jahrhundert», in Schlieben-Lange, Brigitte, Fachgespräche in Aufklärung und Revolution, Tübingen, Niemeyer, p. 17-80.

Maitz, Peter (2014), «Kann - soll - darf die Linguistik der Öffentlichkeit geben, was die Öffentlichkeit will? », in Niehr, Thomas (ed.), Sprachwissenschaft und Sprachkritik. Perspektiven ihrer Vermittlung, Bremen, Hempen, p. 9-26.

Niedzielski, Nancy A. /Preston, Dennis Richard (2000), Folklinguistics, Berlin, Mouton de Gruyter.

Paveau, Anne-Marie (2005), «Linguistique populaire et enseignement de la langue : des catégories communes? », Le français aujourd'hui, n 151, p. 95-107.

Quémada, Bernard (ed.) (1970-1972), Bibliographie des chroniques de langage publiées dans la presse française, vol. 1 (1950-1965), vol. 2 (1966-1970), Paris, Didier.

Remysen, Wim (2009), Description et évaluation de l'usage canadien dans les chroniques de langage: contribution à l'étude de l'imaginaire linguistique des chroniqueurs canadiens-français, Québec, thèse de doctorat, Université Laval. 
Remysen, Wim (2005), La chronique de langage à la lumière de l'expérience canadienne-française : un essai de définition, in Bérubé, Julie/Gauvin, Karine/Remysen, Wim (ed.), Les Journées de linguistique. Actes du 18 colloque 11-12 mars 2004, Québec, Centre interdisciplinaire de recherches sur les activités linguistiques, p. 267-281.

Rossi, Fabio (2015), La questione della lingua filmica nei periodici cinematografici tra il 1936 e il 1945, http://ilpe2015.evenement.usherbrooke.ca/PDF/ILPE2_Resumes_VF.pdf. [Sito consultato il 30 maggio 2015]

Santulli, Francesca (2015), «La riflessione metalinguistica sulla stampa italiana: oltre l'epicedio? », Circula: revue d'idéologies linguistiques, n 2, p. 55-75, http://circula.recherche.usherbrooke. ca/2015-numero-2-ita/ [Sito consultato il 30 giugno 2017]

Schmitt, Christian (2001), «Sprachnormierung und Standardsprachen», in Holtus, Günter/Metzeltin, Michael/Schmitt, Christian (ed.), Lexikon der Romanischen Linguistik, Tübingen, Niemeyer, vol. 1.2, p. 482-484.

Schwarze, Christoph (1977), Sprachschwierigkeiten, Sprachpflege, Sprachbewusstsein: das Phänomen der «Chroniques de langage», Konstanz, Universitätsverlag GmbH.

Schwarze, Sabine (2007), «Identità e alterità nella scrittura scientifica. Prolegomeni alla definizione di uno 'stile scientifico romanzo', in Pistolesi, Elena/Schwarze, Sabine (ed.), Vicini/lontani. Identità e alterità nella/della lingua, Frankfurt, Peter Lang, p. 261-274.

Schwarze, Sabine (2017), «Universalità e culturalità: riflessioni su due modelli interpretativi della scrittura scientifica», in Załęska, Maria (ed.), Il discorso accademico italiano: Temi, domande, prospettive, Frankfurt am Main etc., Lang [Études de linguistique, littérature et art 19], p. 7-42.

Sgroi, Salvatore (2010): Per una grammatica «laica». Esercizi di analisi linguistica dalla parte del parlante, Torino, UTET.

Sobrero, Alberto (2006), «Intorno alle lingue della comunicazione scientifica», in Calaresu, Emilia/ Guardiano, Cristina/Hölker, Klaus (ed.), Italiano e tedesco come lingua della comunicazione scientifica, Berlin, LIT Verlag, p. 1-14.

Trabold, Annette (1993), Sprachpolitik, Sprachkritik und Öffentlichkeit. Anforderungen an die Sprachfähigkeit des Bürgers, Wiesbaden, DUV.

Unesco Publishing (2005), Towards knowledge societies, http://unesdoc.unesco.org/images/0014/001418/141843e.pdf [Sito consultato il 30 maggio 2015].

Verrault, Claude (2007), Description du corpus. ChroQué, www.lexique.ulaval.ca/DescriptionChroQue.aspx [Sito consultato il 30 maggio 2015]. 